\title{
Turismo industrial en la Frontera Norte. Una expectativa de desarrollo para Ciudad Juárez, Chihuahua
}

\author{
Tomás J. Cuevas-Contreras* \\ Isabel Zizaldra-Hernández \\ Francisco Bribiescas-Silva \\ Universidad Autónoma de Ciudad Juárez
}

\section{Resumen}

El trabajo explora las condiciones en las cuales se encuentra el pasado arqueológico-industrial en la frontera norte de México. Por su ubicación, relación comercial y turística con los Estados Unidos, Ciudad Juárez, Chihuahua (enclave de la manufactura conocida como maquiladora, desde finales de 1960), es una comunidad geoestratégica. El examen se basa en una aproximación metodológica, cuanti-cualitativa, útil para identificar las posibilidades del desarrollo del turismo industrial en esta ciudad, que posteriormente pueden repetirse en otras comunidades fronterizas. Se diseñó un instrumento valorativo de 21 reactivos que se aplicó a gerentes y directores en seis maquiladoras localizadas en los diferentes parques industriales de Ciudad Juárez.

\section{Palabras clave}

Turismo industrial, frontera norte, desarrollo, Ciudad Juárez, Chihuahua.

Recibido: 12/09/2012 · Aceptado: 05/10/2012 


\title{
Industrial Tourism in the Northern Border. An expectation of development for Ciudad Juarez, Chihuahua
}

\author{
Tomás J. Cuevas-Contreras \\ Isabel Zizaldra-Hernández \\ Francisco Bribiescas-Silva \\ Universidad Autónoma de Ciudad Juárez
}

\begin{abstract}
The paper inquires into the underlying conditions of the archeological-industrial history of the Mexican northern border. Due to its location and its commercial and touristic relations with the United States, Ciudad Juarez, Chihuahua, is considered a geostrategic manufacturing enclave, known as maquiladoras, since the late 60s. The research is based on a quantitative and qualitative methodological approach, which has been useful in identifying the potential of industrial tourism development within the city, later to be replicated in other border communities. An evaluation tool consisting of 21 questions was designed and tested on managers and directors of six maquiladoras located on the industrial districts in Ciudad Juarez.
\end{abstract}

\section{KeY WORDS}

Industrial tourism, northern border, development, Ciudad Juarez, Chihuahua. 


\section{Introducción}

Las tendencias de la actividad turística internacional se han decantado nuevamente por los gustos y las preferencias de los viajeros en el mundo. La globalización ha jugado un papel preponderante; así, el turismo de salud o médico, el motivado por cuestiones religiosas y el relacionado con los vestigios de la industria, ahora conocido como turismo industrial, han tomado parte en las predilecciones de los turistas.

En el caso del turismo industrial, aunque es plenamente reconocido en Europa, el camino en Latinoamérica es incipiente, pero con una proyección a mediano y largo plazos. En México ya existen ejemplos, entre ellos el Parque Fundidora y la Cervecería Cuauhtémoc Moctezuma en la ciudad de Monterrey, Nuevo León, así como el Tequila Tour en el estado de Jalisco, el cual destaca los procesos de elaboración del tequila, de textiles y cerámica de la región. De igual forma, Altos Hornos de México, en la ciudad de Monclova, Coahuila, ofrece visitas guiadas para mostrar sus instalaciones y procesos, lo único que solicita es llenar un formulario en línea. Sin embargo, la tradición de la actividad turística no ha permeado en el dinamismo de la manufactura en el norte del país, a pesar de que la historia se remonta al menos a 40 años de experiencia con cientos de empresas conocidas como maquiladoras en los estados de Baja California, Sonora, Chihuahua, Coahuila, Nuevo León y Tamaulipas. Poco de ello se conoce como un legado, quizá por el empleo ofrecido o las repercusiones en el desarrollo de la región fronteriza, lo cual pudiera ser una contribución para motivar el interés de quienes visitan los estados fronterizos.

Si bien el impacto de la actividad turística se deriva de los contextos de historia y arte; tradiciones y costumbres; creencias, valores y normas; el turismo industrial engloba antiguas fábricas, maquinaria y colonias industriales, así como empresas actuales o viejas instalaciones.

De ahí que la ventana de oportunidad esté presente en la franja fronteriza de México y particularmente en Ciudad Juárez, ya que el potencial para el turismo industrial es visible, pues cuenta con un patrimonio y una herencia no solo aportados por las empresas de manufactura, sino por las viejas actividades realizadas en el pasado en la región, entre ellas el cultivo de algodón o la producción de whisky tipo americano. 


\section{Planteamiento del problema}

El turismo industrial se basa en el interés formativo y patrimonial de la cultura tecnológica: busca procurar el viaje de la curiosidad, como exterioriza Pardo Abad (2004: 9). La idea de patrimonio ha evolucionado a la par que la sociedad. De ser concebido y valorado por criterios puramente estéticos (lo "bello"), ha pasado a incluir todo aquello que sirve de testimonio de una época y que puede ser objeto de estudio para comprender el pasado y reforzar la memoria colectiva. Por ejemplo:

se deduce que para cada país, centro turístico o atractivo no hay una única imagen turística que tenga validez universal, sino tantas como mercados o segmentos de mercado abarquen los objetivos de comercialización. Aunque en la práctica, como no conviene trabajar con una diversidad, lo que debe hacerse es describir los matices que puedan interesar a cada segmento como complemento de la imagen básica [Boullón, 2004: 136-137].

Las ciudades, por lo tanto, son territorio para la creación y el impulso de nuevos espacios industriales y de servicios debido a sus potencialidades de desarrollo y a la capacidad de generar externalidades. De manera que "El espacio de competitividad creado por el proceso de globalización induce a las ciudades a responder estratégicamente a través de iniciativas locales que estimulan los procesos de desarrollo endógeno" (Vázquez y Madoery, 2001: 9). Por su parte, D’Angella y Go (2009: 431) conciben: "As the tourism system context becomes increasingly fragmented and volatile its stakeholders are pressured to adapt collaboration principles to everyday practice, particularly in the planning and marketing areas". A su vez, Tirado Franco (2006) argumenta sobre las ciudades convertidas: "ya que son importantes centros de producción que han logrado desarrollar un espacio turístico en medio de un ambiente hostil hacia los visitantes”. Este entorno con dualidad urbana se caracteriza por la existencia de dos amplias zonas: los sitios turísticos de la ciudad y sus áreas circundantes.

Desde este panorama, como acotan Rosentraub y Joo (2009), se requiere la planificación de la actividad turística basada en el reflejo del destino mediante la emoción, las creencias y las opiniones de los individuos, de tal manera que su viaje les provoque satisfacciones, es decir, no se debe empujar a los visitantes a los atractivos, al contrario, deben ser capturados por sus propios 
intereses. También se debe pretender a constructos turísticos sociopsicológicos, que predispongan al individuo a participar en las actividades de esparcimiento. En consecuencia, es necesario posicionar a actores emprendedores interesados en la cocreación de nuevos paradigmas de recreación, teniendo en cuenta que contribuirán al rescate y definición de la imagen de la ciudad, en este caso, de las comunidades fronterizas, porque en el "entorno podemos encontrar a actores colectivos con una naturaleza y unas funciones muy variadas: pueden ser organismos públicos, privados y civiles; pueden realizar actividades económicas, financieras, educativas, científicas, políticas, etc." (Villavicencio Carbajal y Casalet Ravenna, 2005: 4).

Se debe considerar el papel que desempeñan los ecomuseos al adaptar como museos los restos industriales. El concepto ecomuseo se basa en la idea de convertir un determinado paisaje industrial en un museo, de manera que se establezca una relación recíproca entre la sociedad y el entorno. En algunos casos los establecimientos pueden mantenerse en activo, pero lo más frecuente es que se recuperen tras el cierre para reforzar la identidad de una región y clarificar de forma específica su naturaleza industrial (Geidetur, 2008). En las zonas fronterizas de México-Estados Unidos la entrada de la manufactura ocurre en los años sesenta, y "La integración económica en el caso de la industria se ha desarrollado a través de un intenso comercio intraindustrial, entre los pares binacionales” (Fuentes, Cervera y Peña, 2007: 223).

Ante estos desarrollos históricos y teóricos alrededor del turismo industrial y estos antecedentes importantes en Europa y en Estados Unidos, es deseable cuestionarse sobre los siguientes temas en relación con la franja fronteriza de México y, particularmente, con Ciudad Juárez: ¿existe algún potencial para el turismo industrial fronterizo? ¿Cuál es el patrimonio industrial de la franja fronteriza sobre el cual se puede desarrollar ese potencial? ¿Mediante qué estrategias públicas y privadas es posible el desarrollo económico a través de experiencias y prácticas sostenibles del patrimonio industrial? Estas y otras interrogantes son centrales para considerar la factibilidad de inserción de un ecomuseo fundamentada en la experiencia maquiladora fronteriza del norte de México. 


\section{Justificación}

La industria maquiladora en la frontera norte de México cumple ya casi medio siglo de haber sido concebida por las élites económicas y políticas del área, y casi cuatro décadas de operación. Además, ha pasado ya por cuatro generaciones, desde el mero ensamblaje hasta el diseño y prueba de modelos de productos industriales, y ha generado una vigorosa clase media. Aunque la industria maquiladora ha tenido sus críticos y detractores, ya forma parte de la historia de la franja fronteriza, una historia que es necesario recuperar no solo por razones que parten de la curiosidad histórica, sino porque en sí misma presenta una oportunidad de desarrollo económico postindustrial.

El turismo industrial permitiría entonces conocer el pasado de la maquiladora instalada a lo largo de la frontera México-Estados Unidos desde los años setenta, así como generar una nueva actividad de turismo y servicios alrededor de esta industria. Las aportaciones conceptuales del turismo industrial permitirían además valorar la sucesión de los distintos cambios productivos y técnicos en las últimas décadas, para generar una conciencia colectiva y dar una nueva faz a la ciudad. Teniendo en cuenta que es un turismo delimitado que busca conocer cosas diferentes y que está centrado tanto en los vestigios de los dos últimos siglos como en las visitas a industrias aún en funcionamiento que muestran los procesos de un determinado producto, se puede considerar el potencial turístico de la franja fronteriza como un turismo basado en la historia y como un turismo vivo. Se debe proponer entonces al turismo industrial como una actividad que, además de prometer preservar el pasado arqueológico-industrial de la frontera, impulse a la región fronteriza por nuevos senderos de desarrollo, por ser un proyecto de carácter patrimonial con carga cultural, histórica y simbólica, pero también económica presente.

Las construcciones industriales precisan un tratamiento específico no convencional debido a las características de sus estructuras (figura 1), su localización, su distribución de espacios y a las plantas que un proyecto de esta magnitud requiere. La reutilización y puesta en valor de edificios e instalaciones ya existentes son aspectos tan importantes como impedir su destrucción y desaparición, pues centra los tipos de acondicionamiento, reutilizaciones posibles y el debate de desarrollo socioeconómico haciendo notar la oportunidad del desarrollo endógeno sostenible. 


\begin{tabular}{|c|c|c|c|c|}
\hline & $\begin{array}{l}\text { Primer polígono indus- } \\
\text { trial Intermex (finales de } \\
\text { los años } 70 \text { principios } \\
\text { de los años } 80 \text { ) }\end{array}$ & & & \\
\hline $\begin{array}{l}\text { RCA (Radio Corporation } \\
\text { of America) } \\
\text { Productos electrónicos } \\
\text { Empresa maquiladora de } \\
\text { primera generación }\end{array}$ & $\begin{array}{l}\text { Principios electrónicos, } \\
\text { maderables, fertili- } \\
\text { zantes, refacciones, } \\
\text { frigoríficos, vestuario, } \\
\text { metálicos, zapatillas, } \\
\text { artículos deportivos y } \\
\text { jueves }\end{array}$ & $\begin{array}{l}\text { Manufacturas dedicadas } \\
\text { al ramo electrónico y } \\
\text { autopartes. Maquila- } \\
\text { doras con concepto: } \\
\text { industrial upgrading y } \\
\text { empresa maquiladora } \\
\text { de segunda generación. }\end{array}$ & $\begin{array}{l}\text { Primera maquiladoras } \\
\text { de tercera generación }\end{array}$ & $\begin{array}{l}\text { Compañía sueca } \\
\text { Electrolux }\end{array}$ \\
\hline $1960-1970$ & $1970-1980$ & $1980-1990$ & 10 & 20 \\
\hline $\begin{array}{l}\text { Estilo norteamericano y } \\
\text { requerimientos estable- } \\
\text { cidos por los estadouni- } \\
\text { denses. } \\
\text { Edificios de cristal y }\end{array}$ & $\begin{array}{l}\text { Se poseen salas de } \\
\text { revisión, sala de corte } \\
\text { y acabado, planchas } \\
\text { calcográficas. }\end{array}$ & $\begin{array}{l}\text { Se cuenta con siste- } \\
\text { ma de seguridad y } \\
\text { vigilancia personal y } \\
\text { electrónica. }\end{array}$ & $\begin{array}{l}\text { Se da inicio al uso de la } \\
\text { maquinaria moderna, } \\
\text { tal como herramientas } \\
\text { de control numérico } \\
\text { computarizado y robots. }\end{array}$ & $\begin{array}{l}\text { Planta más grande } \\
\text { de refrigeradores en } \\
\text { el mundo. Mudando } \\
\text { su planta desde } \\
\text { Michigan. }\end{array}$ \\
\hline
\end{tabular}

acero de grandes dimen-

siones con áreas verdes

y estacionamientos

Fuente: Elaboración propia con base en Fuentes (2001), Carrillo y Hualde (1996) y Cobos González (2005).

Figura 1. Línea de tiempo maquiladora en Ciudad Juárez, Chihuahua

\section{Objetivo general}

Explorar el potencial de desarrollo económico regional de Ciudad Juárez, Chihuahua, mediante la recuperación del patrimonio industrial como una oferta de turismo cultural no centrada en las artes, sino en la cultura tecnológica, asociada al desarrollo de la industria en la franja fronteriza, en particular en las ciudades que comparten un pasado industrial inmediato.

\section{Objetivos específicos}

Identificar el potencial de turismo industrial y cultural de Ciudad Juárez y su ventaja en la frontera.

Examinar el desarrollo económico a través de experiencias y prácticas sostenibles del patrimonio industrial. 


\section{Marco referencial}

El cuidado del patrimonio industrial comenzó en Estados Unidos, primero en el sector privado y luego reorganizado por el sector público con la creación en 1978 del Heritage Conservation and Recreation Service. La actividad estadounidense, sin embargo, se caracteriza por la creación de campañas de estudio realizadas por equipos interdisciplinares y porque en general la conservación se deja a la iniciativa privada. También en Japón esta disciplina cuenta con cierta solidez; en 1977 se fundó la Sociedad de Arqueología Industrial (Casado Galván, 2009), en gran parte porque el interés se encauza mediante una nueva disciplina: la arqueología industrial. Escritores como Donald Dudley o Michael Rix comenzaron a usar el término y para 1966 la arqueología industrial fue adoptada por la universidad inglesa de Bath.

El patrimonio industrial es el más joven de todos los patrimonios porque abarca un conjunto de estructuras, piezas y máquinas que han sido utilizadas en muchos casos hasta fechas recientes (Pardo Abad, 2004: 9). Fábricas, minas, industrias textiles, cervecerías, vapores y cavas despiertan la curiosidad y el interés por visitar elementos patrimoniales tanto de la época industrial como de centros de producción en activo. En este contexto, España trabaja desde hace años para transformar la oferta vacacional y le ha incorporado el turismo industrial. De igual manera, el turismo industrial se ha constituido en un modo de viaje cultural que en Reino Unido, Francia, Alemania, Finlandia y Suecia es una alternativa muy robustecida.

Esta tendencia comenzó a imponerse en Francia a finales de los años cuarenta, cuando las empresas abrieron sus puertas al público con el objetivo de mostrar su cara más moderna. La planta de Peugeot en Sochaux y la cervecería Kronenbourg en Estrasburgo fueron de las primeras compañías en exhibir sus instalaciones. En 1978, la mina de sal de Wieliczka (Polonia) fue el primer emplazamiento industrial declarado patrimonio de la humanidad. Su proximidad a Cracovia favoreció el desarrollo de las visitas turísticas desde comienzos del siglo xix. Actualmente 800000 turistas visitan cada año estas minas, que en un recorrido de 3.5 kilómetros muestran estatuas, cámaras y capillas esculpidas por los mineros en la sal, un lago subterráneo y exposiciones que ilustran su historia (Geidetur, 2008). También armadoras de aviones como la Air Bus, en Toulouse, en el sur de Francia, y Boeing, en Seattle, en el estado de Washington, 
Estados Unidos, ofrecen recorridos para mostrar los procesos y la tecnología en que se basa la construcción de aviones de última generación.

Ahora bien, el impacto psicológico y los fenómenos de transculturación forman parte del imaginario de los destinos. Desde esta dimensión, Holert y Terkessidis (2009: 224) indican: "La imaginibilidad de una sociedad y de una ciudad también es siempre la base del control policial y socioeconómico de sus habitantes". Y extienden la observación: "Una posibilidad de dar una faz a las ciudades es convertirlas en productos visuales, es decir, visibles y gráficos, y consiste en organizar a su población y sus entornos construidos sobre ejes diferenciadores como indígena/foráneo o autóctono/étnico”.

Los fenómenos que se producen con el turismo fronterizo se significan por sus consecuencias, tanto en el campo estricto de la transculturación como en el sociológico, psicológico y económico (Romero, 1977: 138). No obstante:

Las carencias del país, los recursos aun inexplorados, el medio cultural y el conjunto de los problemas sociológicos y psicológicos, determinan en México un nivel de vida comparativamente bajo en la relación con el estadounidense. Aquel presenta grandes diferencias en sus tipos de extremos y éste, en cambio, hace esas distancias muy cortas entre los diferentes segmentos de la estructura socioeconómica de la nación. Alguien ha dicho que el amor del estadounidense por los trajes pintorescos, los desfiles vistosos, la algarabía de las convenciones, etc., son compensaciones de su estandarización. Para el mexicano esa tendencia a cortarlo todo con la misma tijera -construcción de casas, modas, automóviles, etc.- influye para que también se estandaricen los cerebros, las maneras de sentir, las emociones y hasta las ambiciones [Romero, 1977: 144].

Desde esta óptica es necesario considerar que "La recuperación evita la destrucción sistemática de las muestras de nuestro pasado industrial. Las pérdidas, en cualquier caso, han sido numerosas por la incomprensión mantenida durante demasiado tiempo hacia este patrimonio y a la especulación ejercida en el ámbito urbano" (Pardo Abad, 2004: 12).

En este sentido, los impactos del turismo sociocultural se encuentran ligados al respeto mutuo y la amistad de los pueblos (cuadro 1). McCabe (2005: 88) argumenta que: "Early theoretical development of the study of the 'tourist' focused on identifying and defining the nature of the experience of tourists. In so doing, theorists tended to define these experiences in broad relation, or opposition, to other types of experience”. En cambio: 
Estos fenómenos matizan, pues, las actitudes del turista estadounidense al visitar México y explican sus reacciones ante los sentimientos y prejuicios del mexicano. Pero lo importante no es solamente esta explicación genérica, suscitadora de nuevos puntos de vista y probables discusiones, sino el hecho de que el turista, aun el de modesto bagaje de cultura y a pesar de sus conformaciones y deformaciones nacionalistas, es a quien se debe que, poco a poco, vayan disminuyendo estas tensiones de intensa fuerza afectiva entre los dos pueblos [Romero, 1977:145].

\section{Cuadro 1. Principales impactos socioculturales del turismo}

\section{Tipo de impacto}

Historia y arte

Tradiciones y costumbres

Creencias, valores y normas

\section{Consecuencias y potenciales}

- Preservación y rehabilitación de monumentos, edificios y lugares históricos

- Creación de museos de interés cultural (como los museos de la historia de la ciudad)

- Revitalización de formas de arte tradicional: música, literatura, teatro, danza, etc.

- Revitalización de las tradiciones locales: artesanía, festivales, folclore, gastronomía, etc.

- Revitalización de lenguas minoritarias

- Mercantilización extrema de las tradiciones locales

- Intercambio cultural entre visitantes y residentes

- Cambios en valores sociales y creencias religiosas

- Cambios en la estructura económica y roles sociales

- Aumento de problemas sociales: crimen, prostitución, etc.

Fuente: Ayuso y Fullana (2002: 24).

En conjunto, el turismo industrial abarca antiguas fábricas, maquinaria y colonias industriales, además de las empresas actuales o los museos adecuados en viejas instalaciones, por ejemplo:

Ironbridge Gorge Museum. Conjunto de diez museos en torno a la reconstrucción de un pueblo típico de la época victoriana, con sus pozos de hulla, forjas y ladrillares. El pueblo inglés de Ironbridge fue la cuna de la Revolución Industrial. 
Ecomuseo de Bergslagen. Contempla 49 enclaves, desde minas, altos hornos y fundiciones hasta casas de trabajadores, ferrocarriles y centrales de energía. Medio millón de turistas lo visitan cada año en Suecia.

Museo de la Ciencia y de la Técnica de Cataluña. Fue inaugurado en 1984 en una antigua fábrica textil: el Vapor Aymerich, Amat i Jover, de Tarrasa (Barcelona). Su objetivo es acercar al público el patrimonio industrial, científico y tecnológico.

Ecomuseo Creusot-Montceau. Emplazado en una vieja región metalúrgica y minera de La Borgoña, engloba una mina de carbón a cielo abierto, viviendas obreras, un museo de fósiles, una escuela y el Château de la Verrerie, que contiene el Museo del Hombre y de la Industria (Geidetur, 2008).

Las rutas temáticas (cuadro 2) comprenden corredores con amplia cobertura territorial. De acuerdo con la propuesta de Fernández Fuster (1985: 151): “Los núcleos receptores se distribuyen por la geografía mundial de un modo arbitrario. Hay zonas que no conocen el Turismo. Otras en cambio, están saturadas. Los núcleos, como los seres vivos, pueden pasar por varias fases: A) sin Turismo; B) en nacimiento; en crecimiento; en saturación; en disolución”.

\section{CUADRo 2. Ejemplos de rutas temáticas}

\section{Ruta Características}

Ruta minera del

Rhin (Alemania)

Ruta de la lana de Piamonte (Italia)

Ruta del papel (Cataluña)
- Se corresponde con la cuenca del río Emscher y forma un corredor de 800 km, entre las ciudades de Duisburg y Bergkamen. Destacan las minas de carbón de Zollverein, Maximilian y Zollern y el gasómetro de Oberhausen, de $117 \mathrm{~m}$ de altura, convertido en una de las salas de exposiciones más espectaculares de Europa.

- Conecta las ciudades de Biella y Borgosesia a lo largo de 50 km, en los que se evoca el nacimiento de la industria textil. La Fábrica de la Ruta constituye el punto central de este recorrido.

- Localizada al oeste de la provincia de Barcelona, entre los ríos Anoia y Llobregat, contempla la visita del Molino Papelero de Capellades, el de Munné y la moderna fábrica Stora Enso de Castellbisbal, especializada en la producción de papel reciclado. 
De este modo, la reflexión de Homobono resulta sugestiva porque considera que el turismo industrial es una alternativa de desarrollo local (figura 2), que potencia la autoestima de la sociedad local y se incardina en una dinámica de reencantamiento del mundo asociada a la patrimonialización cultural. En particular: "Su puesta en valor requiere una labor paralela de pedagogía de la población local y de sensibilización hacia los valores patrimoniales” (Homobono, 2007: 7-9).

De acuerdo con el modelo, se propone la integración del ámbito proveedor y el de la demanda, por medio de la interacción con el atractivo. Por un lado, el proveedor (industria/negocios) considera el destino, las motivaciones y los sectores, no privado, público y privado. Por otro, la demanda (mercado) es razonada por la motivación (por negocio o familiar) de los viajeros y turistas en la decisión de viaje (destino) (figura 2).

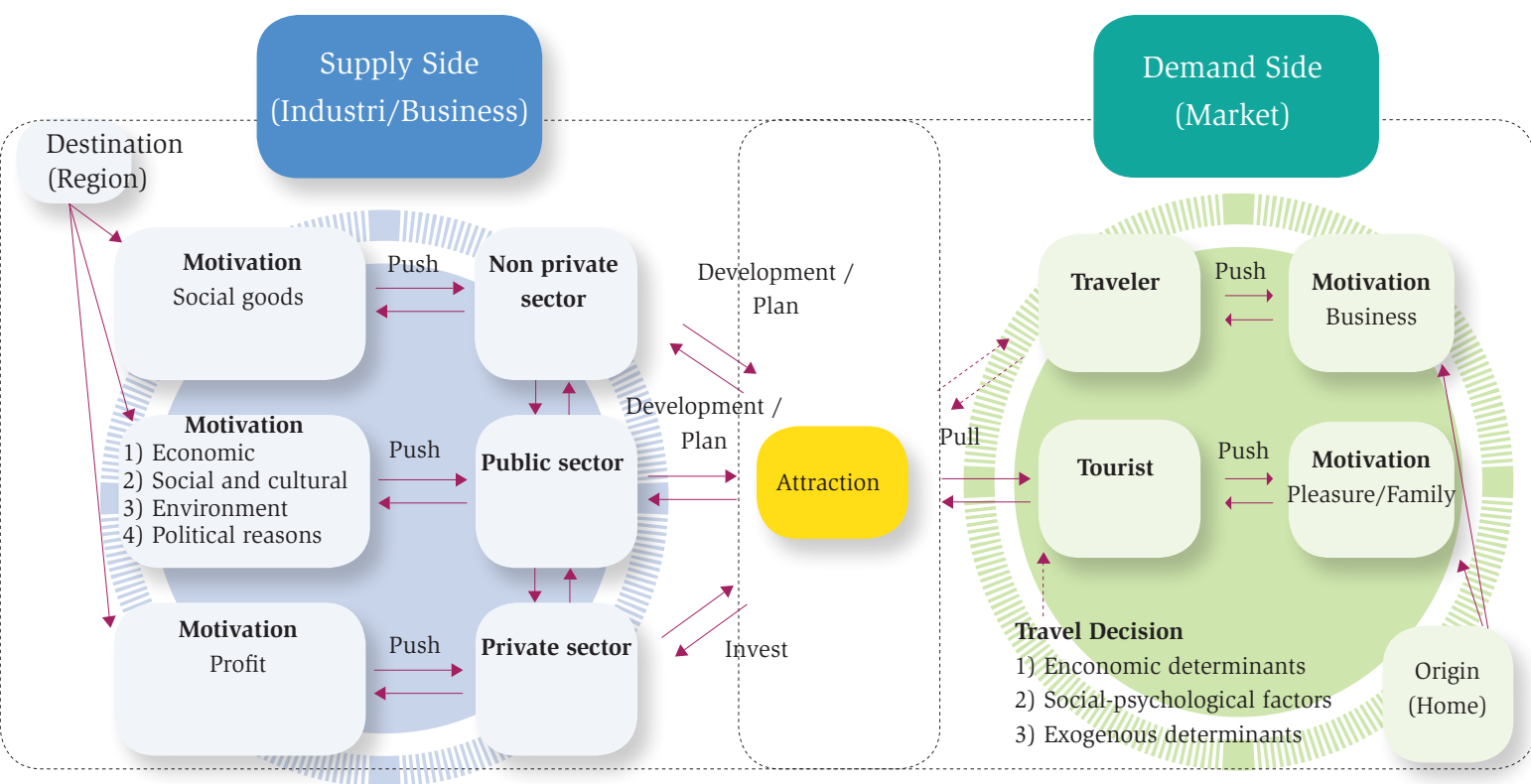

Fuente: Rosentraub y Joo (2009: 762). 


\section{Hipótesis de trabajo}

Una vez adquirido el conocimiento sobre cómo conservar el patrimonio industrial se plantea:

- H1. El patrimonio industrial es significativo para el turismo regional en cuanto dinamizador del desarrollo económico fronterizo de Ciudad Juárez.

- H2. La franja fronteriza norte de México cuenta con profundas experiencias de manufactura/producto para la dinámica del turismo industrial y cultural.

- H3. El concepto de ecomuseo sobre el desarrollo de la manufactura México-Estados Unidos impulsará las expectativas de visita/estadía a las comunidades.

\section{Metodología}

Cuestionario y entrevistas semiestructuradas que permiten un análisis crítico/ procesual para definir las dimensiones de la realidad sociocultural y solo en escasa medida la historia oral. Para ello se desarrolló un instrumento de medición que se aplicó a los actores de la empresa manufacturera en Ciudad Juárez, Chihuahua, principalmente a corporativos de nivel internacional. El cuestionario constaba de 21 preguntas y se desarrolló a través de entrevistas personales (15) en el sector de manufactura. De las 21 preguntas se seleccionaron 15 para el análisis del documento y se agruparon en: a) conocimiento y percepción del turismo industrial; b) implementación del turismo industrial; y c) caracterización del turismo industrial.

\section{Resultados}

De los resultados de este primer acercamiento exploratorio a través de una muestra no probabilística es importante destacar que el instrumento se aplicó a gerentes y directores locales. Entre las empresas encuestadas en el área de manufactura se encuentran Valeo, Delphi, ADC, Strattec, ABACo y Lexmark. Esta fase experimental aporta las percepciones e ideas que se tienen en torno a la tendencia del turismo industrial en Ciudad Juárez.

Es conocido que las empresas de manufactura, independientemente de la existencia de recorridos formales, constantemente llevan a cabo recorridos dentro de sus instalaciones para mostrar a sus visitantes -clientes potenciales, 


\section{Cuadro 3. Conocimiento y percepción del turismo industrial}

\begin{tabular}{|c|c|c|}
\hline 1. ¿Está usted informado acerca de lo que es el turismo industrial? & Total & $\%$ \\
\hline a) Sí & 12 & 80 \\
\hline b) No & 3 & 20 \\
\hline Gran total & 15 & 100 \\
\hline $\begin{array}{l}\text { 2. ¿Considera que la población juarense mostrará interés por el turis- } \\
\text { mo industrial? }\end{array}$ & Total & $\%$ \\
\hline
\end{tabular}
a) Sí

$\begin{array}{lcr} & 11 & 73 \\ & 4 & 27 \\ \text { Gran total } & 15 & 100 \\ \text { indus- } & \text { Total } & \%\end{array}$
3. Cuál será un factor determinante para el éxito del turismo industrial?

a) El interés público

b) El apoyo del gobierno

$\begin{array}{rr}2 & 13 \\ 0 & 0 \\ 5 & 33 \\ 8 & 53 \\ 0 & 0\end{array}$

c) La alianza de las empresas maquiladoras

d) Todas las anteriores

Gran total $15 \quad 100$

4. Qué beneficios considera que traería el turismo a Ciudad Juárez

Total

$\%$

a) Generaría nuevas fuentes de empleo

320

b) Atraería capital extranjero

213

c) Cambiaría la imagen de la ciudad

0

d) Todas las anteriores

$9 \quad 60$

e) No traerá ningún beneficio

f) Otro

Gran total 15

5. ¿Cuál sería una razón para no implementar el turismo industrial en Total Ciudad Juárez?

a) Generaría más pérdidas que ganancias $\quad 2 \quad 13$

b) El estado socioeconómico en el que se encuentra la ciudad $\quad 77$

c) No habría una respuesta por parte de la gente $\quad 4 \quad 27$

d) Otro $\quad 2 \quad 13$

Gran total $15 \quad 100$

Fuente: Trabajo de campo

clientes formales y proveedores- sus procesos e instalaciones en forma detallada. En este contexto las operaciones en las maquiladoras ya cuentan con un camino avanzado y en algunos casos con personal enfocado al desarrollo de esta actividad. Sin embargo, es de subrayar la reticencia de algunas empresas que argumentan contar con procesos únicos y en algunos casos delicados. 
En Ciudad Juárez existen 324 empresas maquiladoras, las cuales se encuentran catalogadas de la siguiente forma: automotriz, $29 \%$; electrónicos, $18 \%$; plásticos y metales, $9 \%$; empaque, $9 \%$; eléctrico, $7 \%$; médico, $5 \%$; call center, $3 \%$; otros, $20 \%$ (ver Redco). Cabe resaltar que no todas cuentan con procesos delicados o únicos. Por tal motivo, las empresas más susceptibles en su inclusión son las de arneses, vestiduras para autos y call centers, entre otras. Sin embargo, las propuestas son finalmente analizadas y consensuadas en el corporativo de las empresas (cuadros 3, 4 y 5)

\section{CUADRo 4. Implementación del turismo industrial}

\begin{tabular}{|c|c|c|}
\hline $\begin{array}{l}\text { 6. ¿Estaría dispuesto a participar en una feria de turismo industrial en } \\
\text { la ciudad? }\end{array}$ & Total & $\%$ \\
\hline a) Sí & 14 & 93 \\
\hline b) No, ipor qué? & 1 & 7 \\
\hline Gran total & 15 & 100 \\
\hline $\begin{array}{l}\text { 7. ¿Estaría dispuesto a dar a conocer sus procesos de producción con } \\
\text { un fin lucrativo? }\end{array}$ & Total & $\%$ \\
\hline a) Sí & 5 & 33 \\
\hline b) No & 10 & 67 \\
\hline Gran total & 15 & 100 \\
\hline $\begin{array}{l}\text { 8. ¿El recibir un apoyo por parte del gobierno sería un factor decisivo } \\
\text { para promover el turismo industrial en su empresa? }\end{array}$ & Total & $\%$ \\
\hline a) Sí, ¿por qué? & 9 & 60 \\
\hline b) No, ipor qué? & 6 & 40 \\
\hline Gran total & 15 & 100 \\
\hline 9. ¿Cómo implementará el turismo industrial su empresa? & Total & $\%$ \\
\hline $\begin{array}{l}\text { a) Organizando visitas a la empresa para dar a conocer los procesos de } \\
\text { producción y la historia de la empresa }\end{array}$ & 4 & 27 \\
\hline b) Creando un museo dentro de la empresa & 2 & 13 \\
\hline c) Haciendo alianzas con otras empresas para organizar ferias & 8 & 53 \\
\hline d) Otro_ & 1 & 7 \\
\hline
\end{tabular}

Fuente: Trabajo de campo 


\section{Cuadro 5. Caracterización del turismo industrial}

10. ¿A qué público estaría enfocado el recorrido en su empresa?

a) Niños de primaria

b) Adolescentes de secundaria y preparatoria

c) Profesionistas con experiencia en el ramo

d) Estudiantes universitarios

e) Público en general

$\begin{array}{ccc} & \text { Total } & \% \\ & 0 & 0 \\ 1 & 7 \\ 4 & 27 \\ \text { Gran total } & 15 & 100 \\ & 7 & 20 \\ & \text { Total } & \% \\ & 2 & 13 \\ & 12 & 80 \\ & 1 & 7 \\ & 0 & 0\end{array}$

11. ¿Cuánto considera que sería un buen tiempo para el recorrido?

Gran total $15 \quad 100$

12. ¿Cuánto cobraría por un recorrido en su empresa?

Total $\%$
a) $\$ 50$ a $\$ 100$
b) $\$ 101$ a $\$ 200$
c) 201 a 300
d) Otro

\begin{tabular}{|c|c|c|}
\hline & & \\
\hline & 7 & 47 \\
\hline & 1 & 7 \\
\hline & 0 & 0 \\
\hline & 7 & 47 \\
\hline Gran total & 15 & 100 \\
\hline & Total & $\%$ \\
\hline & 4 & 27 \\
\hline & 5 & 33 \\
\hline vernirs & 4 & 27 \\
\hline & 2 & 13 \\
\hline Gran total & 15 & 100 \\
\hline resa? & Total & $\%$ \\
\hline & 5 & 33 \\
\hline & 5 & 33 \\
\hline & 0 & 0 \\
\hline & 0 & 0 \\
\hline & 5 & 33 \\
\hline Gran total & 15 & 100 \\
\hline & Total & $\%$ \\
\hline & 6 & 40 \\
\hline & 2 & 13 \\
\hline & 7 & 47 \\
\hline & 0 & 0 \\
\hline Gran total & 15 & 100 \\
\hline
\end{tabular}

\section{3. ¿Qué incluiría el recorrido?}

a) Una visita guiada solamente

b) Una visita guiada con un souvenir de regalo

c) Una visita guiada y al final del recorrido ofrecer una tienda de souvernirs

d) Otro

Fuente: Trabajo de campo 


\section{Discusión de resultados}

Es de destacar la existencia de viejos procesos que han caído en desuso por parte de la maquiladora y la propuesta de conformar un museo relacionado con la evolución de la manufactura. El estudio se centra en los procesos para procurar la activación patrimonial y la legitimación de identidades de los vestigios de la industria y la manufactura en Ciudad Juárez, en términos de proyección de futuro y en cuanto a los agentes, interesados en comprometerse o no con actuaciones en el desarrollo del turismo industrial, percibidos como intereses y expectativas involucradas (gráfica 1). En este contexto, Homobono (2007: 7-9) señala el ámbito académico, socioeconómico y geográfico:

Académico. Las experiencias son muy diversas y casi todas pasan por la musealización. Es la manera más extendida de conservar y usar un patrimonio valioso pero sin el carácter de pieza única y excepcional. Así, se otorga al patrimonio industrial un interés especial que despierta la conservación y la reutilización con fines turísticos y didácticos: es básico mostrarlo en todas sus dimensiones y hacerlo comprensible a la sociedad actual. De modo que su puesta en valor requiere una labor paralela de pedagogía de la población local y de sensibilización hacia los valores patrimoniales.

Socioeconómico. El turismo industrial es una alternativa de desarrollo local, potencia la autoestima de la sociedad local y considera la dinámica de reencantamiento del mundo asociada a la patrimonialización cultural.

d) Otro

c) No habrá respuesta por parte de la gente en el que se encuentra la ciudad

a) Generaría más pérdidas que ganancias b) El estado socioeconómico

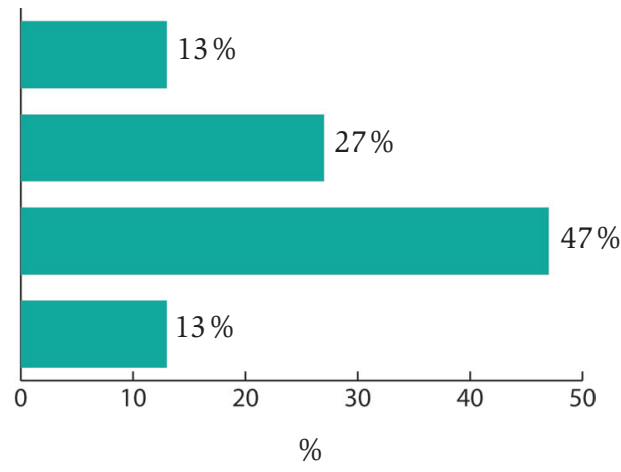

Fuente: Elaboración propia con base en el trabajo de campo. 
Geográfico. Los esfuerzos de un ecomuseo deben dirigirse a aumentar el desarrollo económico del área geográfica concreta, impulsando la atracción y el interés turístico para los visitantes. Así pues, la idea es fundamentar un futuro a partir del pasado y buscar el compromiso y la responsabilidad de los habitantes locales.

En la gráfica 1 las respuestas reflejan que $47 \%$ de los encuestados considera que la razón para no implementar el turismo industrial en Ciudad Juárez es el estado socioeconómico en el que se encuentra la ciudad. El resultado muestra una poca intención de inversión industrial en estas empresas debido a la crisis de manufactura global, principalmente de Estados Unidos, y a la situación de seguridad, pues han invertido mucho en el rubro de seguridad interna. Abrirse al turismo industrial generaría mayores controles en la entrada a las empresas por parte de los visitantes, aunque existe la intención de apoyar y generar opciones relativas al turismo industrial. Por tal motivo se propone:

a) Crear museos donde se exponga la evolución de distintos productos a lo largo de la historia. Desde la llegada de la industria maquiladora a Ciudad Juárez, la variedad de productos que se fabrican ha evolucionado a través de los años. Sería un aspecto interesante y de mucho enriquecimiento conocer su transformación en el tiempo. Estos museos se pueden ubicar como una extensión de las instalaciones de la empresa o bien en un lugar independiente de esta.

b) Implementar corredores dentro de las instalaciones de la empresa. Estos corredores servirán para que la gente visite las instalaciones de la empresa y pueda observar el proceso de transformación de los productos, creando en el público una expectación y un nuevo interés por la industria local.

c) Crear ferias de turismo industrial. Programar una o dos veces al año este tipo de ferias donde participen distintas empresas maquiladoras de la localidad. En estos eventos se montarían locales donde la gente pudiera interactuar en los distintos procesos productivos que se llevan a cabo, propiciando que se aprecie la industria de una manera distinta.

Respecto a la pregunta ¿cómo implementaría el turismo industrial en su empresa? (gráfica 2), 53 \% respondió que haría alianzas con otras empresas para 
organizar ferias. Aquí sobresale el interés de las organizaciones industriales en desarrollar planes de vinculación con universidades locales, aprovechando su estructura humana y física y, además de lograr la colaboración entre los organismos industriales y cámaras correspondientes para la exposición histórica de sus productos. Las diferentes esferas de gobierno también mostraron interés en organizar ferias, convenciones y museos movibles, donde resaltarían las aportaciones de logística, experiencia y fomento de la cultura local.

d) Otro

c) Haciendo alianzas con otras empresas para organizar ferias

b) Creando un museo dentro de la propia empresa

a) Organizando visitas a la empresa para dar a conocer los procesos de producción y la historia de la empresa

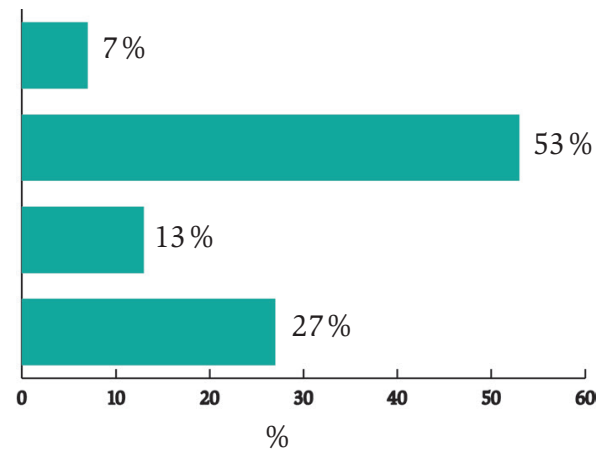

Fuente: Elaboración propia con base en el trabajo de campo.

GrÁFICA 2. ¿Cómo implementaría el turismo industrial en su empresa?

A la pregunta ia qué público estaría enfocado el recorrido de su empresa? (gráfica 3), 47 \% respondió que al público en general. Esto promueve los corredores internos para conocer las áreas de procesos y sus productos, pues llevarían a los visitantes a tener un ángulo de observación adecuado, además de ser un corredor seguro, sin riesgos de accidentes. Se detectó que el objetivo mayor sería para todo público, sin embargo, se deduce un alto porcentaje involucrado con estudiantes universitarios y profesionistas.

Además, esta participación activa del público en general deberá ser apoyada con las diferentes alianzas para fomentar e incrementar la cultura local en pro de una proyección de imagen a esta ciudad. Los empresarios industriales están considerando la afluencia de visitas en un ganar-ganar, es decir, desplegar la historia industrial de su organización y de la realización de un nuevo nicho de negocios. 
e) Público en general

d) Estudiantes universitarios

c) Profesionistas con experiencia en el ramo

b) Adolescentes de secundaria y preparatoria

a) Niños de primaria

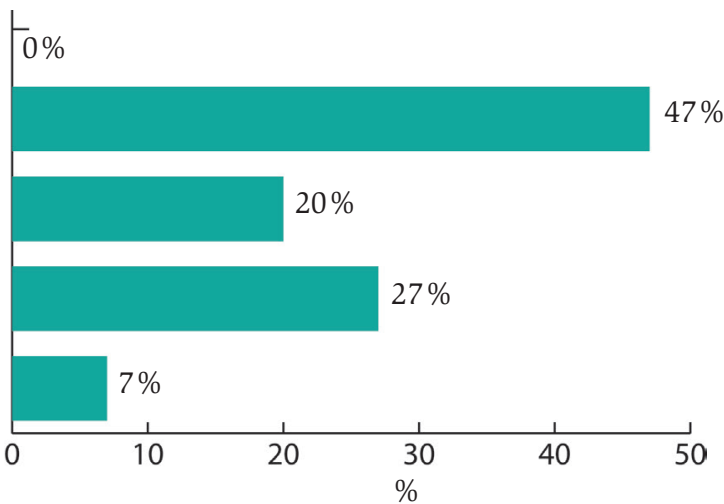

Fuente: Elaboración propia con base en el trabajo de campo.

GráfICA 3. ¿A qué público estaría enfocado el recorrido de su empresa?

d) Otro

c) Abriría posibilidades de crecimiento dentro de la empresa

b) Sería una carga extra innecesaria que sólo generaría estrés

a) Los motivaría a trabajar con más esmero dentro del área de su trabajo

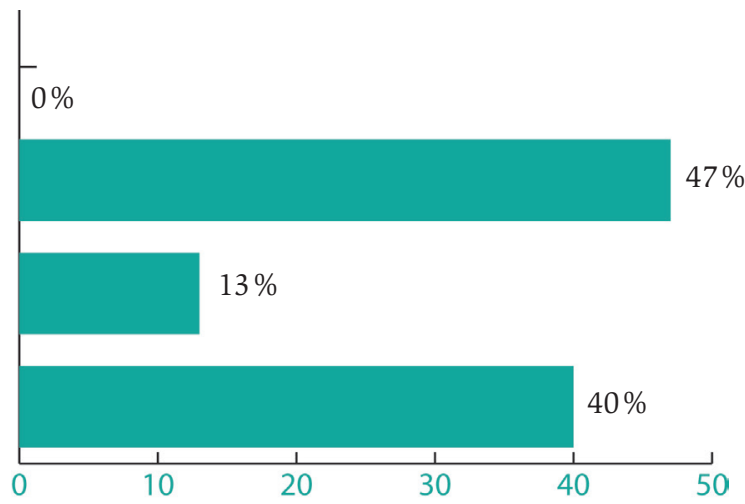

Fuente: Elaboración propia con base en el trabajo de campo.

\section{GRÁfICA 4. ¿Cómo impactaría un turismo industrial a sus empleados?}

La respuesta al cuestionamiento de icómo impactaría el turismo industrial a sus empleados? (gráfica 4) fue mayoritariamente (47 \%) que abriría posibilidades de crecimiento dentro de la empresa. Con base en un incremento de participación en una cultura de la empresa y de la comunidad, el personal de las firmas involucradas mejorará notablemente su desempeño laboral, lo que creará un ambiente más competitivo. 
La implantación del turismo industrial como un nuevo nicho de negocios generaría una estructura básica en materia de logística y mantenimiento, e incluso propiciaría la captación de clientes potenciales; además, a partir de la transparencia de sus procesos y productos, se producirá una cocreación de valor más fuerte entre las firmas y los consumidores. Esto podría promover una estructura humana de atención al público, lo que abre las posibilidades de un crecimiento interno.

\section{Conclusiones}

Es posible definir tres tipos de procesos propicios para el turismo industrial: de manufactura o industrial, comercial productiva, y comercial inactiva. Las características generales para su participación son:

- Industrial/manufactura. Muestran procesos a través de líneas de ensamble o diferentes departamentos productivos. Ejemplos de ello son Delphi, ADC, Strattec, Electrolux, etcétera.

- Comercial/productiva. Exponen el proceso de sus productos desde su ingreso, transformación y presentación final para su comercialización. Ejemplos: Lechería Lucerna, Cervecería Carta Blanca, Embotelladora de la Frontera, Harineras y, sotoleras como 5 Tragos y Don Cuco.

- Comercial/productiva inactiva. Procesos de elaboración y transformación en desuso como Juárez Whisky, Procesadoras de Algodón -en el valle de Juárez-, Cervecería Cruz Blanca, Brandy San Felipe, entre otras.

La declaración de Chávez (2010: 187) permite reflexionar sobre el entorno de Ciudad Juárez: "para distinguir una sociedad de otra se requiere conocer cuáles son los elementos culturales que utilizan, sean estos producto de su cultura, o producidos por otras y asumidos por ellos para transformar el entorno que les rodea en función de sus necesidades y asumirse como parte de ese espacio cultural”. Entonces, la arqueología industrial adquiere singular importancia por la influencia de las empresas de manufactura instaladas a finales de los años sesenta.

El turismo industrial es una derivación del producto y del espacio cultural en el que se ubica. Se identifica con el entorno por su interacción con los procesos 
que le ha tocado vivir tanto a la sociedad como a los actores relacionados. Por tal motivo, las propuestas tienden a la creación de museos, a la implementación de corredores, a la creación de ferias y, en el mejor de los casos, a visitas guiadas en el interior de las empresas. Así, es posible encontrar diferentes casos en cada uno de estos rubros, de ahí que se proponga un museo relacionado con la manufactura y la incorporación de recorridos en empresas representativas en procesos de arneses, vestiduras e inyección de plásticos, entre otros.

Por último, el detonador son los visitantes, una vez que eligen un destino. El lugar, por su parte, inicia sus actividades incorporando la hotelería, las comunicaciones y la propaganda (Fernández Fuster, 1985). La actividad del turismo industrial en la frontera México-Estados Unidos será sin lugar a dudas pionera, aunque es cierto que el concepto se conoce "genéricamente", pero aún falta el consenso con los actores e interesados, como expectativa de desarrollo para Ciudad Juárez, Chihuahua.

\section{Recomendaciones}

El escrutinio exploratorio sobre el turismo industrial ha permitido considerar los parques industriales dentro de su contexto histórico y patrimonial para Ciudad Juárez. Su análisis permite especular sobre su incorporación como parte de la oferta turística. De la misma manera, es menester recuperar y documentar los viejos procesos y tecnologías utilizadas en la comunidad, con la finalidad de forjar su impulso, enriquecimiento y conservación.

\section{Agradecimientos}

A Priscila Rosario Ortiz Candia, Silvia Margarita Baca Rodallegas, Diana Sáenz Chávez y Mayra Evelina Palma Domínguez, por su apoyo en el trabajo de campo. 


\section{Fuentes consultadas}

Ayuso, Silvia y Pere Fullana (2002). Turismo sostenible. Barcelona: Rubes.

Bianchi, Raoul (2007). "Tourism and the Globalisation of Fear: Analysing the Politics of Risk and (in) Security in Global Travel”. Tourism and Hospitality Research, 7, 64-74.

Boullón, Roberto (2004). Marketing turístico: una perspectiva desde la planificación. Buenos Aires: Ediciones Turísticas.

Carrillo, Jorge y Alfredo Hualde (1996). "Maquiladoras de tercera generación. El caso de Delphi-General Motors". Journal of Borderlands Studies.

Casado Galván, I. (2009). "Breve historia de la protección del patrimonio industrial”. Contribuciones a las Ciencias Sociales [en línea], noviembre de 2009. Disponible en: www.eumed.net/rev/cccss/06/icg4.htm

Chávez, Jorge (2010). Entre rudos y bárbaros: construcción de una cultura regional en la frontera norte de México. México: El Colegio de Chihuahua (Miradas).

Cobos González, Carmen (2005). “Inaugura Electrolux planta de refrigeradores”. Las buenas noticias también son noticia [en línea]. Disponible en: http://fox.presidencia.gob.mx/buenasnoticias/?contenido $=19250 \&$ [2010, 25 de febrero].

D’Angella, Francesca y Frank M. Go (2009). “'Tale of Two Cities' Collaborative Tourism Marketing: Towards a Theory of Destination Stakeholder Assessment”. Tourism Management, 30, 429-440.

Fernández Fuster, Luis (1985). Enciclopedia de Turismo. México: Nueva Editorial Interamericana.

Fuentes, César (2001). "Los cambios en la estructura intraurbana de Ciudad Juárez, Chihuahua, de monocéntrica a multicéntrica”. Frontera Norte, 13 (25), enero-junio, 95-118.

Fuentes, César M., Luis Cervera y Sergio Peña (2007). "La integración económica entre México-Estados Unidos y su impacto en el sistema urbano espacial de una región transfronteriza: Ciudad Juárez-El Paso”. Nóesis. Revista de Ciencias Sociales y Humanidades, 16, enero-junio, 206-232. Geidetur (2008). "Turismo industrial, el viaje de la curiosidad”. Savia [en línea], junio, 48-51. Disponible en: http://www.uhu.es/GEIDETUR/archivos/48_savia59_industrial.pdf 
Homobono, José I. (2007) "El patrimonio industrial y sus activaciones: turismo, museos, eco-museos y reutilización”. Kobie. Antropología Cultural, 12, 5-33.

------ (2008). "Del patrimonio cultural al industrial: una mirada socioantropológica”, en X. Pereiro, S. Prado y H. Takenaka (coords.). Patrimonios culturales: Educación e interpretación. Cruzando límites y produciendo alternativas [en línea]. Universidad del País Vasco, 57-74. Disponible en: http://www.euskomedia.org/PDFAnlt/antropologia/11/12/12057074. pdf

Kaczynski, Andrew y John L. Crompton (2004). “An Operational Tool for Determining the Optimum Repositioning Strategy for Leisure Service Departments”. Managing Leisure [en línea], 9, julio, 127-144. Disponible en: http://agrilife.org/cromptonrpts/files/2011/06/2_2_6.pdf

Kliot, Nurit y Yoel Mansfeld (1999). "Case Studies of Conflict and Territorial Organization in Divided Cities”. Progress in Plannning [en línea], 52, 167-225. Disponible en: www.elsevier.com

McCabe, Scott (2005). “'Who is a Tourist?’: A Critical Review”. Tourist Studies, 5 (1), 85-106.

Pardo Abad, Carlos J. (2004). "La reutilización del patrimonio industrial como recurso turístico. Aproximación geográfica al turismo industrial”. Treballs de la Societat Catalana de Geografía, 57, 7-32.

Romero, Héctor Manuel (1977). Sociopsicología del Turismo. México: Daimon. Rosentraub, Mark S. y Mijin Joo (2009) "Tourism and Economic Development: Which Investments Produce Gains for Regions?” Tourism Management, 30, 759-770.

Ryan, Richard M. y Edward L. Deci (2000). "Self-Determination Theory and the Facilitation of Intrinsic Motivation, Social Development, and WellBeing”. American Psychologist, 55 (1), 68-78.

Tirado Franco, Roberto (2006). "Conceptualización de la estructura urbana de una ciudad turística”. VIII Congreso Nacional y $2^{\circ}$ Internacional de Investigación Turística de Sectur, 14 al 16 de junio de 2006. Monterrey, Nuevo León.

Vázquez, Antonio y Oscar Madoery (comps.) (2001). Transformaciones globales, instituciones y políticas de desarrollo local. Rosario: Homo Sapiens. 
- Turismo industrial en la Frontera Norte. Una expectativa de desarrollo para Ciudad Juárez, Chihuahua

Villavicencio Carbajal, Daniel Hugo y Mónica Casalet Ravenna (2005). “La construcción de un 'entorno' institucional de apoyo a la industria maquiladora en la frontera norte de México”. Revista Galega de Economía, 14, junio-diciembre, 1-20.

\section{Páginas electrónicas}

www.ecomusee-creusot-montceau.fr

www.ekomuseum.se

www.ironbridge.org.uk

www.mnactec.cat

www.virtualtourist.com 